\title{
Connecting Information to Promote Public Health
}

\begin{abstract}
With the development of information technology in the past 12 years, China has established the specialized or vertical web-based information systems for data collection of disease and related risk factor. These information systems are described as public health information systems (PHIS) in China. When PHIS has thus evolved in a haphazard and fragmented way under the pressure administrative, economic and legal, local health workers who are overburdened with multiple and timeconsuming reporting requirements cannot deliver timely, accurate and complete data. Seeing that the information of disease surveillance is provided by hospitals and health service centers in China and that the development of electronic medical record (EMR) and electronic health record (EHR) occurs under the policy and investment of health since 2009 in China, there should be a connection and data sharing mechanism between EMR (EHR) and PHIS to support public health surveillance and public health decision-making. The paper aims at discussing current status and problems of PHIS in China, making a brief introduction on the blueprint of health information technology in China, exploring solution for interoperability between PHIS and EHR (EMR), and sharing some experiences and lessons from pilot project on automatic notifiable infectious disease reporting.
\end{abstract}

Keywords: public health, information system, data exchange, China

\section{Introduction}

Public health surveillance is the continuous systematic collection, analysis and interpretation of health-related data needed for the planning, implementation, and evaluation of public health practice. Such surveillance can serve as an

Manuscript received August 1, 2016; accepted October 30, 2016

Xue-mei Su ( $\varangle)$, Jia Zhao

National Center for Public Health Surveillance and Information Service, Chinese Center for Disease Control and Prevention, Beijing 102206, China

Email: suxm@chinacdc.cn early warning system for impending public health emergencies, document the impact of an intervention, or track progress towards specified goals, and monitor and clarify the epidemiology of health problems, in order to set priorities and inform public health policies and strategies (WHO, 2015a, 2015b). Surveillance draws on many data sources - these are both population-based (such as mortality surveillance in sentinel populations) and institution-based (such as disease in sentinel health facilities) (WHO, 2008). With the development of information technology in the past 12 years, China has established the specialized or vertical web-based information systems for data collection of disease and related risk factor. These information systems for surveillance are described as public health information systems (PHIS) in China.

A main purpose of building PHIS is to provide timely, complete, regular and quality information for disease surveillance which is a common public service in all countries (Turbelin \& Boëlle, 2013; WHO, 2015a, 2015b). When PHIS has thus evolved in a haphazard and fragmented way under the pressure of administration, economics, legal or donation, local health workers who are overburdened with multiple and time-consuming reporting requirements cannot deliver timely, accurate and complete data. Seeing that the information of disease surveillance is provided from hospitals and health service centers in China and the development of electronic medical record (EMR) and electronic health record (EHR) occurs under the policy and investment of health since 2009 in China, there should be a connection and data sharing mechanism between EMR (EHR) and PHIS to support public health surveillance and public health decision-making (Knaup, et al., 2006). It is a fundamental requirement to implement efficient national PHIS. As a result, automated data exchange between PHIS and EMR (EHR), and interoperability of the two systems are of extreme importance.

This paper aims at discussing the current status and problems of PHIS in China and making a brief introduction on the blueprint of health information technology (IT) in China, exploring a solution for interoperability between PHIS and EMR (EHR), and sharing some experiences and lessons from a pilot project on automatic notifiable infectious disease reporting. 


\section{Public health infrastructure}

The public health infrastructure in China has adopted a four-tier public health system: national, provincial, city or prefecture and county levels. All the centers for disease control and prevention (CDC) at local levels come under the leadership of the local health authority, while the national CDC is under the leadership of National Health and Family Planning Commission of China, which is formerly called the Ministry of Health (MOH) and it has only a technical assistance role. All medical care providers, such as hospitals, community or township health service centers, village clinics, and other health facilities like blood stations, also have responsibilities on disease control (Figure 1). By the end of April 2015, the total number of medical and health facilities had been 98,500, with 3,500 CDCs, 26,000 hospitals, 34,000 community health service centers and 37,000 township health service centers (National Health and Family Planning Commission of the Peoples Republic of China, 2015).

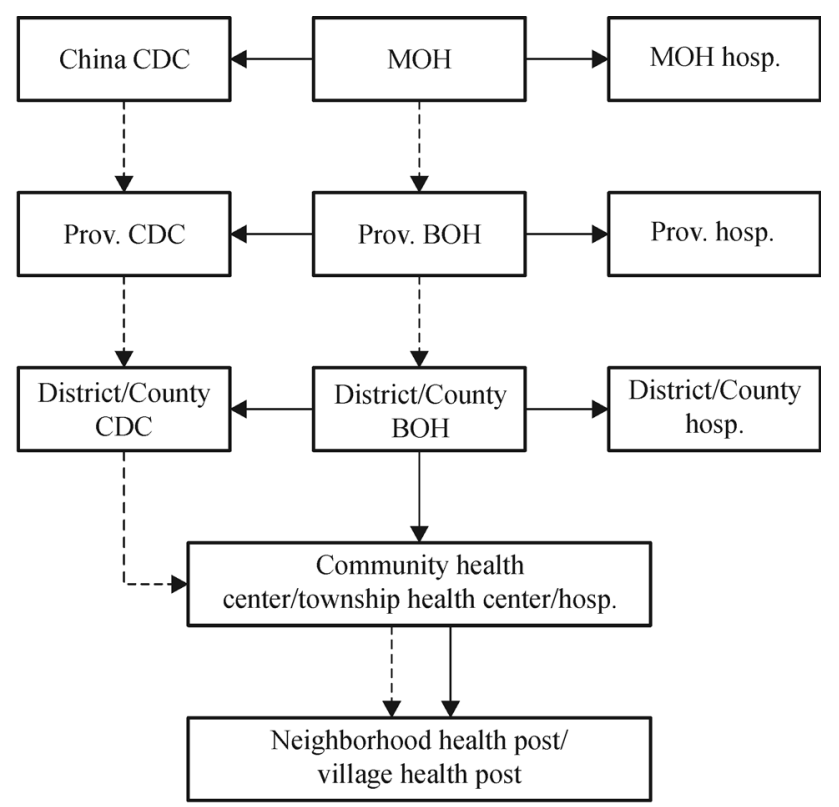

- Administration relationship

- - - - - Technical assistance

Figure 1. Organization \& Management of Public Health.

\section{Health IT in China}

Generally speaking, there are three stages in health IT in China (Figure 2). The development of PHIS is mainly in the second stage.

\subsection{Development of PHIS}

The SARS crisis that occurred in 2003 revealed gaps in China's infectious disease control. Many deficiencies in

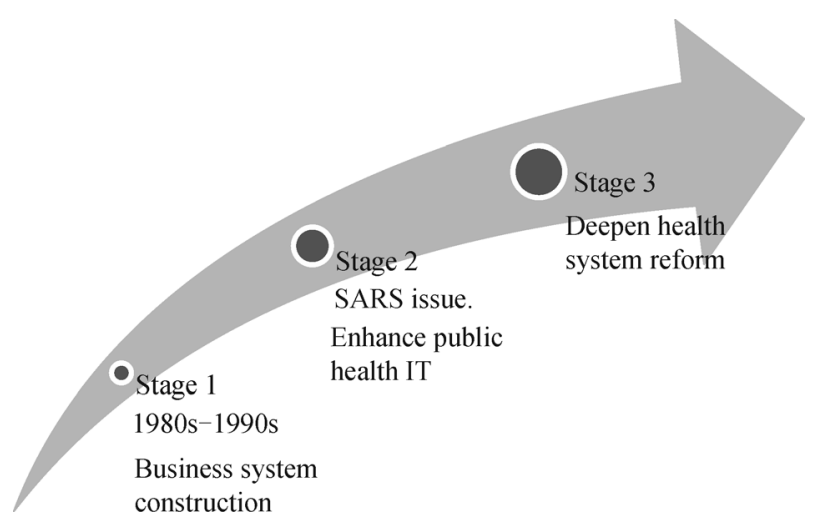

Figure 2. Three stage of health IT in China.

infectious disease surveillance (IDS) were identified, such as poor timeliness and unavailability of original individual case data in the CDCs at city level and above. The Chinese government has strengthened the investment in the public health system. With the financial support, National CDC has gradually developed web-based PHIS. So far, PHIS in China includes:

1) Case-based surveillance information system such as notifiable infectious disease reporting, enhanced specific infectious disease surveillance (such as TB, AIDS/HIV, and influenza, birth defect) and death surveillance (sentinel health facilities), children vaccination, adverse events following immunization, occupational disease reporting, non-communicable disease surveillance (sentinel health facilities).

2) Event-based surveillance information system, such as public health emergency surveillance system.

3) Sample-based surveillance information system, such as drinking water and vector surveillance.

4) Facility-based management information system (MIS), such as MIS on personnel, infrastructure, equipment, and finance of all levels of CDCs, laboratory MIS and vaccine and cold chain MIS as well.

The efficient PHIS have urgent demand on the rapid availability of data collection. As a key component of PHIS, national notifiable infectious disease reporting system (NNIDRS) is required to be aware of the occurrence and outbreak of infectious diseases as early as possible (Liu, et al., 2008). Therefore, the 39 notifiable diseases in China are divided into class A, B and C. Class A includes plague and cholera, which are required to be reported within 2 hours. Class B, including HIV/AIDS, viral hepatitis, poliomyelitis, human infection with avian influenza, H7N9 influenza, measles, and Class C, including influenza, mumps, rubella, acute Hemorrhagic conjunctivitis, leprosy, are required to be reported within 24 hours. The data of NNIDRS comes from various sources, including public health agencies (all levels CDCs), medical care providers, other health facilities like blood stations. To date, there are more than 68,000 users in the system 
including hospitals, health service centers, CDCs and health administrative departments at all levels and about 20,000 infectious disease cases reported each day.

In China, medical care providers who have access to the internet can report the cases through the internet to NNIDRS, although some providers have EMR with automatic infectious disease report functional module. For the providers who have no access to the internet, they send reports by mail, telephone, or facsimile to a local CDC for data entry. The workflow for notifiable infectious disease reporting is as follows. If a notifiable infectious case is diagnosed, the doctor in charge of the patient will fill in the paper-based infectious disease reporting cards or make supplementary entry on the patient's information which is required by infectious disease report through EMR (EHR) and then report the case to the department responsible for infectious disease management. The department will check the information and report the case into NNIDRS. Local CDC will audit, revise, conduct an analysis and disseminate the report, and the local CDC will make an epidemiological investigation when it is necessary.

Advances in information communication and technology have largely promoted the development of IDS, and have facilitated data collection, management, analysis and information dissemination. In addition, the timeliness, completeness and sensitivity of infectious disease surveillance have substantially increased. Now, there are six main functions in the system. That is, web-based real-time data collection, level-based information management, online data analysis, retrieval and sharing, integrated GIS for outbreak detection, dynamic quality evaluation and timely information feedback. The timeliness of IDS has increased to $96.96 \%$, and the average time interval between diagnosis to notification, which was more than 4 days 10 years ago, is now 13.6 hours, (Wang, Guo, Y., Guo, Q., \& Zhang, 2011). Concurrent with the improvement of IDS data quality, surveillance data analysis, feedback and dissemination have been strengthened in China. NNIDRS provides data analysis which is compiled into daily, weekly, monthly and yearly reports, which are available at NNIDRS for authorized users. The national summary data is also available on the websites of the former MOH and CDC. Historical surveillance data can be queried at the website of the center for public health science. NNIDRS also provides statistical tables and all the statistical data can be visualized in a map through embedded GIS (Geographic Information System) tools. Through the computing model of the system, an infectious disease cluster can be identified.

Technology assists IDS practitioners to continually build their capacity for emergency response. For instance, the Wenchuan earthquake in 2008 damaged 566 reporting sites in total. The requirement was evident to monitor and assess potential disease outbreaks in earthquake affected areas. China CDC was able to adapt an innovative informatics tool which called cell phone-based reporting system to recover infectious disease reporting. The tool had been designed originally to collect syndrome data in earthquake affected area and submit data in the form of SMS message to NNIDRS (Guo \& Su, 2012). Another example of rapid, innovative adoption of surveillance technology was the development of the China Infectious Disease Automated alert and Response System (CIDARS) in 2005. Its development was based on the existing data from NNIDRS on 28 diseases that are outbreak-prone and require prompt action, and on methods of fixed-threshold detection, temporal detection, and spatial detection to detect aberration. During the pilot testing period, CIDARS generated 308 signals, involving nine diseases, 69 (22.4\%) of which were identified as possible positive signals that triggered further field investigation, with nine cholera outbreaks confirmed (Yang, et al., 2011).

\subsection{Problems of PHIS}

In spite of 12 years of PHIS's operation, some problems still exist. The first one is the problem of paper-based reporting system. In China, many vertical PHIS have been developed and launched by a variety of agencies or programs. Reliable and useful PHIS mainly depend on original data reporting from medical care providers. However, the fragmented and poorly coordinated systems require excessive data entry. Therefore, medical care workers are overburdened and fail to complete the paper forms. As a result, timely, accurate and complete data for surveillances cannot be collected. The second problem arises from EMR (EHR)-based reporting. Although some hospitals and health service centers have had EMS and EHR, the connection and data sharing mechanisms between PHIS and EMR (EHR) are not sufficient enough to support diseases surveillance and help public health decision making.

\subsection{Third stage in health IT in China}

With the development of EMR and EHR under the policy and investment of health since 2009 in the third phase of Figure 2, PHIS has been criticized as vertical information system in recent years in China. To better understand the specialized or vertical problem of PHIS, the development of the third stage of health IT needs to be described briefly.

In the third stage, the Chinese government released its ambitious secondary round of national health reform plan in 2009. The overall goals of the plan include establishing a sound basic health care system covering urban and rural residents and providing safe, effective, convenient, inexpensive medical and health services. Health IT has been recognized as one of the eight support pillars necessary to achieve the goals of health reform.

Former MOH has provided the top level design of health IT. In the future, national, provincial and regional health information platform will be established in China. In 
addition, six main applications, such as public health, will get information from EMR- and EHR-based regional health information platform (RHIP). RHIP information comes from hospitals, health service centers, and public health services agencies. One important factor is to facilitate the implementation of the uniform health card for residents. Meanwhile, information standard and security guarantee system must be considered (Figure 3).

The acknowledgement of the importance of Health IT in China's health reform plan has stimulated policy that supports Health IT investment. As one paper in BMC Medical Informatics and Decision Making described (Lei, Sockolow, Guan, Meng, \& Zhang, 2013), the Central Government allocated $\mathrm{CNY} 9.5 \times 10^{10}$ (approximately $1.5 \times 10^{10}$ USD) in 2011 as part of the health reform package to promote the use of Health IT in hospitals in China. This investment exceeded the total investment in HIT over the past 30 years. Investments by $\mathrm{MOH}$ included a pilot program to implement EMRs in 97 hospitals in 2010 and in additional 92 hospitals in 2011. Provincial level trial programs for regional health exchange were supported and all county level medical care providers in China's central western provinces were each financed by about $\mathrm{CNY}$ $2 \times 10^{6}$ to acquire hardware and software for implementing Health IT. Each provincial government and hospital also added investment to EMR. Meanwhile, the former $\mathrm{MOH}$ initiated policies to promote Health IT and established a standards bureau which issued a series of Health information standards. Some of the EHR or EMR related standards are listed as follows: EMR basic architecture and data standards released in 2009, EMR Basic Regulations released in 2010, EMR system Functional Profiles released in 2010, Technical profiles of EMR-based Hospital
Information Platform, and Technical profiles of EHRbased regional Health Information Exchange Platform.

These policy and financial support greatly accelerated the adoption of EMR and EHR in China. EMR refers to a system which manages medical records for clinical purposes. According to a 2012 national survey in China, nearly $50 \%$ of 1,004 responding hospitals had adopted the basic forms of EMR (48.18\%) (China Hospital Information Management Association, 2013). In China, it has been nearly 26 years since hospital information system were first adopted in Chinese hospitals. At present, most medical care providers have their own hospital management information system. Some of them have developed or are developing EMR, expand LIS plus PACS. Much of the information collected in these systems is valuable for public health. On the other hand, in China, EHR refers to systems that manage longitudinal health data for the population. EHR includes basic personal information, summary on major diseases and health issues, and major health service records. In the future, it is desirable to realize medical and health care across institutions and across regions through EHR with the residents' health card. It also makes medical treatment and health management convenient throughout the individual's lifetime. However, there is still a long way to implement EHR completely in China, because of the lack of awareness and investment of local government and health facilities.

\section{Pilot project on interoperability between PHIS and EMR (EHR)}

With the investment of EMR and EHR, parts of EMR and

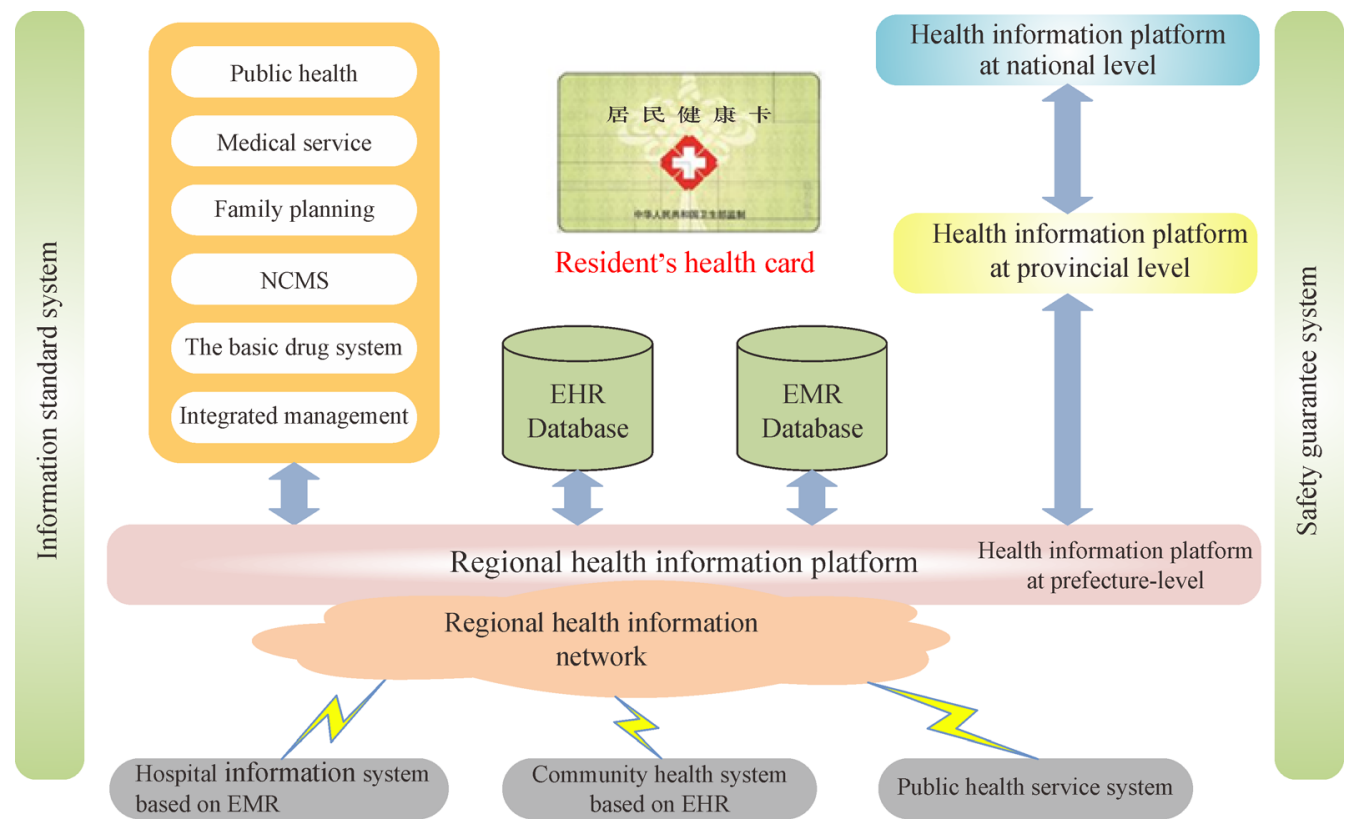

Figure 3. Top level design of health IT in China. 
several EMR- and EHR-based regional health information platforms in China have been developed due to public health information demands. But how to interoperate PHIS outside the EMR (EHR) turns out to be a big challenge. National CDC has carried out a pilot project on notifiable infectious disease case records (NIDCR) between PHIS and hospital information system.

Although HL7 has been effective in communication, it is not practicable in Chinese hospitals because there is no technical support for data translation and exchange in HL7 messaging format in China. On the other hand, nearly all hospitals in China can easily access web technologies, including HTML, browsers, Java, JavaScript and web server, which will support exchanging health information in XML format on the internet. A solution based on the technology which binds Message Oriented Middle ware (MOM) and Web Services is presented after several modes for implementing reliable Web Service are analyzed.

Based on the above technologies of data exchange, China CDC is launching a pilot project. Until now, data exchange of Yinzhou district of Ningbo city has been implemented in the developed Zhejiang province, which has developed better EMR and EHR-based RHIP that is recognized as a good example of RHIP in China. Meanwhile, HIS-based data exchange in one hospital of Guangdong province has also been conducted. Now, data exchange project with Shanghai health platform are being carried out.

In the implementation of data exchange, five priority areas should be considered to achieve interoperability in health care applications: patient identifiers, semantic interoperability, data interchange standards, core data set and data quality (Byrne et al., 2014; Hammond, Bailey, Boucher, Spohr, \& Whitaker, 2010; Lenz, Beyer, \& Kuhn, 2007; Müller, Ückert, Bürklec, \& Prokosch, 2005). The XML schema structure has been studied, including its content and semantics, case information expressed with XML document, message construction and transmission such as data extraction, secure message transfer and message parsing.

\section{Experience and lessons learned}

With the absence of a unique identifier, records could only be matched by relying on a combination of different kinds of information from the patient record such as name, sex, date of birth, race, or identity card of residents. But these methods were imperfect, and they did not always result in accurate matching. Unique identifiers are needed both for the benefit of each individual and for creating databases to evaluate and monitor population health (Byrne, et al., 2014; Hammond, Bailey, Boucher, Spohr, \& Whitaker, 2010).

Although China $\mathrm{MOH}$ has issued a set of health information standards since 2010, PHIS and most of HIS applied in China had been developed before the standards were issued, and it was very hard to adapt to these systems at later stage.

The problem was guaranteeing the consistent use of the standards. In some cases, it was a matter of awareness of the existence of applicable standards. In other cases, there was a lack of understanding of the role of standards. Overlapping and competing standards still existed.

Unnecessary data collection could overwhelm an information system. So, to define core data set of public health data at the national level would lead to more efficient communication of public health surveillance (WHO, 2008).

A solution based on the technology binding MOM and Web Services can be used to implement EMR or EHRbased automatic reporting to PHIS, greatly reducing the PHIS data entry burden on health workers at the medical care providers.

It is necessary to improve the quality of data entry at the facility level. If the worker understand the importance of the data and how they would be used, collecting and entering them accurately and completely would be seen as more important. Therefore, the worker collecting data in medical care providers became as important as the person using the data (Hammond, Bailey, Boucher, Spohr, \& Whitaker, 2010; WHO, 2008).

Local Health Bureaus were responsible for EHR- and EMR-based RHIP's construction. But local CDCs were only recognized as data users, their participations in data design were inadequate. As a result, data was not utilized well. Therefore, successful implementation of RHIP depends on the participation of the local CDCs.

China is a large country with 32 provinces. Due to the different economic levels, health IT performance, a large number of health facilities of each province, data exchange of national PHIS can be put priority on connecting to RHIP or provincial health platform, not to each hospital and health service center.

\section{Conclusions}

To conclude, effective PHIS require timely access to all health data from all sources. In most parts of the world today, these data are more likely to come from many different and unconnected systems - but must be organized into a composite whole. Next important steps for public health IT managers are to consider how to solve the issues on patient identifier, semantic interoperability, data interchange standards, core data set and data quality in China so as to implement efficient PHIS connecting to EMR or EHR (Hammond, Bailey, Boucher, Spohr, \& Whitaker, 2010). With the implementation of blueprint of health IT in China, data exchange of national PHIS can put priority on connecting to RHIP or provincial health platform, not to each hospital and health service center. 


\section{References}

Byrne, C.M., Mercincavage, L.M., Bouhaddou, O., Bennett, J.R., Pan, E. C., Botts, N.E., Olinger, L.M., Hunolt, E., Banty, K.H., \& Cromwell, T. (2014). The Department of Veterans Affairs' (VA) implementation of the Virtual Lifetime Electronic Record (VLER): findings and lessons learned from Health Information Exchange at 12 sites. International Journal of Medical Informatics, 83, 537-547.

China Hospital Information Management Association. (2013). Survey of health information technology adoption status among Chinese hospitals, 2011-2012. Retrieved from http://www.chima.org.cn/ index.php? $\mathrm{m}=$ content $\& \mathrm{c}=$ index $\& \mathrm{a}=$ show \&catid $=10 \& \mathrm{id}=145$.

Guo, Y., \& Su, X.M. (2012). Mobile device-based reporting system for Sichuan earthquake-affected areas infectious disease reporting in China. Biomedical and Environmental Sciences, 25, 724-729.

Hammond, W.E., Bailey, C., Boucher, P., Spohr, M., \& Whitaker, P. (2010). Connecting information to improve health. Health Affairs (Millwood), 29, 284-288.

Knaup, P., Garde, S., Merzweiler, A., Graf, N., Schilling, F., Weber, R., \& Haux, R. (2006). Towards shared patient records: an architecture for using routine data for nationwide research. International Journal of Medical Informatics, 75, 191-200.

Lei, J., Sockolow, P., Guan, P., Meng, Q., \& Zhang, J. (2013). A comparison of electronic health records at two major Peking University Hospitals in China to United States meaningful use objectives. BMC Medical Informatics and Decision Making, 13, 96104.

Lenz, R., Beyer, M., \& Kuhn, K.A. (2007). Semantic integration in healthcare networks. International Journal of Medical Informatics, 76, 201-207.
Liu, D., Wang, X., Pan, F., Xu, Y., Yang, P., \& Rao, K. (2008). Webbased infectious disease reporting using XML forms. International Journal of Medical Informatics, 77, 630-640.

Müller, M.L., Ückert, F., Bürkle, T., \& Prokosch, H.U. (2005). Crossinstitutional data exchange using the clinical document architecture (CDA). International Journal of Medical Informatics, 74, 245256.

National Health and Family Planning Commission of the People's Republic of China. (2015). The number of Chinese medical and health institutions at the end of April in 2015. Retrieved from http:// www.nhfpc.gov.cn/mohwsbwstjxxzx/s7967/201506/9e460a735 34b42e3b34968ee20e4160f.shtml

Turbelin, C., \& Boëlle, P.Y. (2013). Open data in public health surveillance systems: a case study using the French Sentinelles network. International Journal of Medical Informatics, 82, 10121021.

Wang, L., Guo, Y., Guo, Q., \& Zhang, C. (2011). Evaluation on quality of internet-based reporting of notifiable communicable diseases in China, 2005-2008. Disease Surveillance, 25, 912-914.

WHO. (2008). Framework and standards for country health information systems. 2nd ed. World Health Organization.

WHO. (2015a). Integrated disease surveillance programme. Retrieved from http://www.who.int/csr/labepidemiology/projects/surveillance/ en

WHO. (2015b). Public health surveillance. Retrieved from http://www. who.int/topics/public_health_surveillance/en/

Yang, W., Li, Z., Lan, Y., Wang, J., Ma, J., Jin, L., Sun, Q., Lv, W., Lai, S., Liao, Y., et al. (2011). A nationwide web-based automated system for outbreak early detection and rapid response in China. Western Pacific Surveillance and Response Journal: WPSAR, 2, 10-15. 